\title{
In Vitro Transdifferentiation of Human Fetal Type II Cells Toward a Type I-like Cell
}

\author{
CHERIE D. FOSTER, LINDA S. VARGHESE, RACHEL B. SKALINA, LINDA W. GONZALES, AND SUSAN H. GUTTENTAG \\ Department of Pediatrics, University of Pennsylvania School of Medicine, The Children's Hospital of Philadelphia, Philadelphia, \\ Pennsylvania 19104
}

\begin{abstract}
For alveolar type I cells, phenotype plasticity and physiology other than gas exchange await further clarification due to in vitro study difficulties in isolating and maintaining type I cells in primary culture. Using an established in vitro model of human fetal type II cells, in which the type II phenotype is induced and maintained by adding hormones, we assessed for transdifferentiation in culture toward a type I-like cell with hormone removal for up to $144 \mathrm{~h}$, followed by electron microscopy, permeability studies, and RNA and protein analysis. Hormone withdrawal resulted in diminished type II cell characteristics, including decreased microvilli, lamellar bodies, and type II cell marker RNA and protein. There was a simultaneous increase in type I characteristics, including increased epithelial cell barrier function indicative of a tight monolayer and increased type I cell marker RNA and protein. Our results indicate that hormone removal from cultured human fetal type II cells results in transdifferentiation toward a type I-like cell. This model will be useful for continued in vitro studies of human fetal alveolar epithelial cell differentiation and phenotype plasticity. (Pediatr Res 61: 404409, 2007)
\end{abstract}

$\mathrm{T}$ The distal alveolar epithelium contains type I and II cells. Type II cells comprise $75 \%$ of distal lung epithelium, $7 \%$ of the surface area, and produce surfactant. Type II cells have many well-characterized markers, including lamellar bodies, surfactant proteins A, B, C, and D (SP-A, B, C, and D) (1), and the aspartic protease pepsinogen $\mathrm{C}$ (PGC) (2).

Type I cells, although less numerous, cover $93 \%$ of adult lung surface area and provide the gas exchange surface (1). Other type I functions, such as ion transport and fluid homeostasis, are less known. Type I cells form a tight monolayer with low permeability (3). However, the utility of previously described type I biochemical markers (4) has been limited by variable reproducibility and expression between species.

Challenges in isolation and culture have limited progress in type I cell biology. Despite isolated reports $(5,6)$, there are no widespread primary culture models. Instead, rat type II cells transdifferentiated toward a type I cell on tissue culture plastic (7) have served as a proxy for type I cells $(8,9)$. There are limited data with regards to transdifferentiation in adult human

Received August 7, 2006; accepted November 7, 2006.

Correspondence: Cherie D. Foster, M.D., Abramson Research Center 416C, The Children's Hospital of Philadelphia, 3516 Civic Center Boulevard, Philadelphia, PA 19104-4318; e-mail: fosterc@email.chop.edu

NIH grants HL-077266 (C.D.F.) and HL059959 (S.H.G.) supported the research in this proposal.

DOI: $10.1203 /$ pdr.0b013e3180332c6d alveolar epithelium (10), with no previous in vivo descriptions in human fetal lung. Rodent model transdifferentiation and the observation that type II cells serve as progenitors of type I cells after alveolar injury in mature lung (11) have led to the assumption that type I cells are derived from type II cells. However, the possibility that both are derived from a common precursor and retain some degree of plasticity has not been examined in the human fetal lung.

Because of its potential plasticity, fetal alveolar epithelium provides unique opportunities to study differentiation/ transdifferentiation pathway(s) in the developing lung. We previously demonstrated that type II cells from human fetal lung can maintain a differentiated phenotype with dexamethasone, cAMP, and isobutylmethylxanthine (DCI) (12) and that these conditions induce differentiation of type II cells from naive human fetal lung epithelium (13). Here, we demonstrate that DCI withdrawal from cultured human fetal type II cells results in transdifferentiation toward type I-like cells. Transdifferentiation is associated with diminution of type II morphology, decreased expression of type II markers, and induction of type I cell markers. Importantly, transdifferentiated cells behave like type I cells, with decreased permeability and increased transepithelial resistance (TER), indicating a tight type I cell monolayer. Our data establish transdifferentiation as a tool for future study of human alveolar type I cell biology.

\section{METHODS}

Reagents. Dexamethasone, isobutyl methylxanthine, and 8-bromo-cAMP were obtained from Sigma Chemical Co. (St. Louis, MO). Carboxyfluorescein and Texas Red dextran were from Molecular Probes (Eugene, OR). All other reagents were purchased from Fisher (Fair Lawn, NJ), Pierce (Rockford, IL), or Invitrogen (Carlsbad, CA).

Antisera used herein included cytokeratin (DAKO, Carpinteria, CA), Ki-67 (Vision Biosystems, Norwell, MA), vimentin (US Biologicals, Swampscott, MA), zonula occludens-1 (ZO-1) (Zymed, South San Francisco, CA), SP-B (Chemicon, Temecula, CA), caveolin-1 $\alpha$ (Cav -1) (Santa Cruz Biotechnologies, Santa Cruz, CA), claudin 7 (Zymed), SP-A (US Biologicals), PGC (Abcam, Cambridge, UK), glyceraldehyde-3-phosphate dehydrogenase (GAPDH) (Chemicon), and plasminogen activator inhibitor-1 (PAI-1) (BD Transduction Laboratories, Lexington, KY). An epitope-specific pro-SP-C rabbit antiserum (NPRoSP-C; Met ${ }^{10}-\mathrm{Glu}^{23}$ of rat proSP-C) was donated by Dr. Michael Beers.
Abbreviations: Cav-1, caveolin-1; DCI, dexamethasone, cAMP, and isobutylmethylxanthine; GAPDH, glyceraldehyde-3-phosphate dehydrogenase; PAI-1, plasminogen activator inhibitor-1; PGC, pepsinogen C; SEM, scanning electron microscopy; SP-A, SP-B, SP-C, surfactant proteins A, B, C; TEM, transmission electron microscopy; TER, transepithelial resistance; ZO-1, zonula occludens-1 
Cell culture. Human fetal lung from 14- to 18-wk therapeutic abortions came from the Birth Defects Laboratory in the Department of Pediatrics, University of Washington Medical Center (Seattle, WA). All tissue was obtained and handled under protocols approved by the Committee for Human Research, Children's Hospital of Philadelphia.

Fetal lung epithelial cells were prepared as described (13). Cells were cultured on tissue culture plastic for up to $9 \mathrm{~d}$ in $10 \mathrm{nM}$ dexamethasone, 0.1 $\mathrm{mM}$ 8-bromoadenosine- $3^{\prime}, 5^{\prime}$-cyclic monophosphate, and $0.1 \mathrm{mM}$ isobutyl methylxanthine (DCI, or "hormones") to induce and maintain type II cell differentiation. Transdifferentiation was accomplished by hormone withdrawal after the type II cell phenotype was established on $\mathrm{d} 4$.

Epithelial cell viability and purity assays. The LIVE/DEAD Viability/ Cytotoxicity Kit (Molecular Probes) was used to determine cell viability. This two-color fluorescent viability assay relies on the retention of calcein AM in live cells (red) and infiltration of ethidium homodimer (EthD-1) into damaged cells (green). Cytokeratin (to identify epithelial cells) and vimentin (to identify fibroblasts) immunostaining were used to assess epithelial cell purity. Proliferation was characterized by Ki67 immunostaining.

Ultrastructural studies. Transmission electron microscopy (TEM) samples were prepared and imaged as previously described (14), with the addition of polycarbonate filters for cross-sectional images. For scanning electron microscopy (SEM), cells were cultured on glass and subjected to hexamethyldisilazine for critical point drying. Samples were sputter coated with gold palladium target and representative images captured with a Philips XL 20 scanning microscope at $5 \mathrm{kV}$, with a working distance at $100 \mu \mathrm{m}$. All TEM supplies were from Electron Microscopy Sciences (Fort Washington, PA) or Polysciences (Warrington, PA)

Alveolar epithelial barrier studies and indirect immunofluorescence. Barrier properties were assessed by fluorescent dye diffusion measurement across cell monolayers plated on Transwell permeable membranes with $0.4-\mu \mathrm{m}$ pores (Corning, Corning, NY), as detailed elsewhere (15). Filtered fluorophore concentrations were assessed using a SpectraMAX Gemini microplate fluorimeter (Molecular Devices, Sunnyvale, CA). Barrier function was also characterized by measuring TER with an ohmmeter (World Precision Instruments, Sarasota, FL). With all barrier studies, a confluent monolayer with tight junctions was confirmed using indirect immunofluorescence for the tight junction protein ZO-1 (16).

Western immunoblotting. Cells were harvested in lysis buffer (1\% Triton $\mathrm{X}-100,150 \mathrm{mM} \mathrm{NaCl}, 50 \mathrm{mM}$ Tris-HCL, $5 \mathrm{mM}$ ethylenediamine tetraacetic acid, 5\% glycerol, $\mathrm{pH}$ 8.0) mixed with $1 \times$ protease inhibitor (Roche, Indianapolis, IN). Westerns were performed using NuPAGE Bis-Tris gels with MES running buffer (Invitrogen) and transferred to polyvinylidine difluoride membrane. Primary antibodies used were claudin 7, Cav-1, PAI-1, and NProSP-C at 1:1000, SP-A at 1:2500, SP-B at 1:4000, PGC at 1:5000, and GAPDH at 1:20,000. Blots were probed with secondary antibodies conjugated to Alexa Fluor 680 (Molecular Probes) or IRdye 800 (Rockland, Gilbertsville, PA) at a dilution of 1:10,000 for all primary antibodies. Blotted proteins were detected and analyzed using the Odyssey infrared imaging system (Li-Cor, Lincoln, NE).

Real-time reverse transcriptase polymerase chain reaction (RT-PCR). Total cellular RNA was isolated with RNA STAT-60 Reagent (Tel-Test, Friarswood, TX). Purity was verified by the OD 260:280 ratio. Integrity was screened using the eukaryote total RNA nano assay on an Agilent 2100 bioanalyzer (Agilent, Palo Alto, CA). Real-time RT-PCR reactions using a singleplex strategy were performed using an ABI Prism 7900 system [Applied Biosystems (ABI), Foster City, CA]. The two-step PCR protocol was performed as detailed elsewhere (2). All reagents/probes were obtained from ABI. Fluorescence intensity was recorded during the annealing step of each cycle. The following primer/probe sets, as listed on the ABI Web site (http://www.allgenes.com), were used: SP-A1 Hs00831305, SP-C Hs00161628, SP-B Hs00167036, PGC Hs00160052, claudin 7 Hs00600772, Cav-1 Hs00184697, aquaporin $5 \mathrm{Hs} 00387048$, PAI-1 Hs00167155, and GAPDH Hs99999905. All assays were determined to be in the linear amplification range by using cDNA standards created from RNA from alveolar epithelial cells cultured for $4 \mathrm{~d}$ in DCI (type II cells) or from banked frozen adult lung tissue (type I cells).

Statistical analysis. Results are given as mean \pm standard error of the mean (SEM). $t$ tests (for lamellar body quantification) or analysis of variance (ANOVA) (all other studies) were performed using GraphPad Prism 4.00 for Macintosh (GraphPad, San Diego, CA). All protein and RNA results were normalized to GAPDH.

\section{RESULTS}

Cell viability and proliferation. We examined cell purity, proliferation, and viability in differentiating ( 44 and 7 in DCI) and transdifferentiating ( $4 \mathrm{~d}$ DCI followed by $3 \mathrm{~d}$ Waymouth's) culture conditions (three experiments, five random fields per condition). Cell cultures were $\geq 91 \%$ epithelial cells by cytokeratin and vimentin immunostaining. No epithelial cells were proliferating, as indicated by absent Ki67 staining. There were no differences in cell viability between groups (4d DCI: $94 \pm 0.4 \%, 7 \mathrm{~d}$ DCI: $92.6 \pm 1.0 \%, 4 \mathrm{~d} \mathrm{DCI} / 3 \mathrm{~d}$ Waymouth's: $92.0 \pm 1.0 \%, p=$ not significant).

Morphologic studies. Effects of transdifferentiation on morphology were examined by TEM and SEM. Naive epithelial cells exhibited no lamellar bodies. By $4 \mathrm{~d}$ in DCI (4 d DCI), lung epithelial cells demonstrated hallmarks of differentiated type II cells, including lamellar bodies and microvilli (Fig. $1 A, C)$, as previously noted (13). SEM supported the TEM phenotype, with microvilli visible as abundant, short cytoplasmic projections (Fig. 1E). Transdifferentiating cells demon-
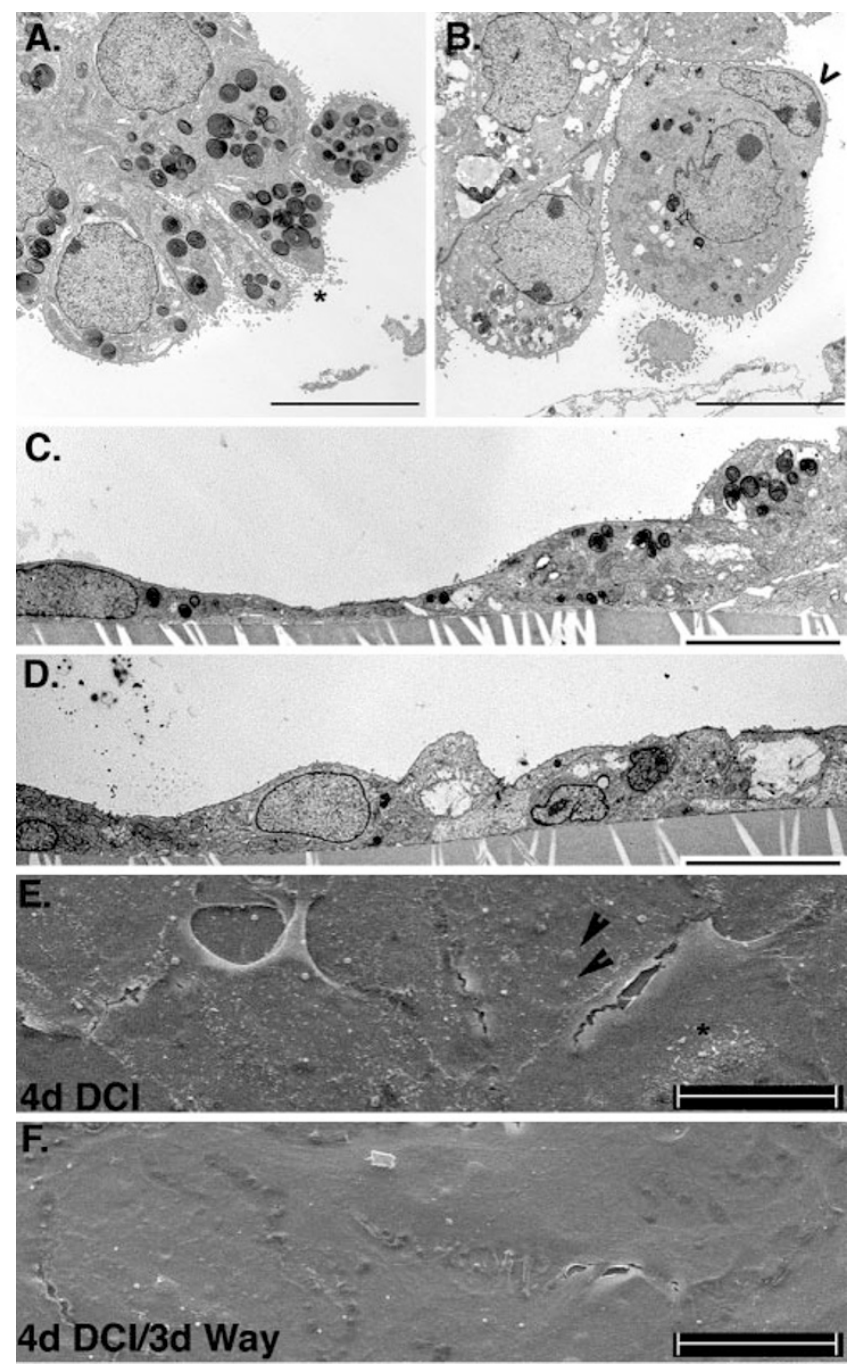

Figure 1. Transdifferentiation via hormone withdrawal alters type II cell morphology and surface characteristics. Representative electron micrographs of human fetal lung epithelial cells treated with DCI for $4 \mathrm{~d}$ (4 d DCI) $(A, C, E)$, or $4 \mathrm{~d}$ DCI followed by $72 \mathrm{~h}$ without hormones (4 d DCI/3 d Waymouth's) $(B, D, F)$. TEM of cell pellets $(A, B)$ and cross-sectional slices of plated cells $(C, D)$ at $\times 5000$. SEM images of plated cells $(E, F)$ at $\times 1000$. $(A, E)$ : Microvilli indicated by asterisk. (B) Blunting of microvilli indicated by open arrowhead. $(E)$ Lamellar bodies indicated by bold arrowhead. $(A-D)$ Bars = $10 \mu \mathrm{m} .(E, F)$ bars $=20 \mu \mathrm{m}$. 

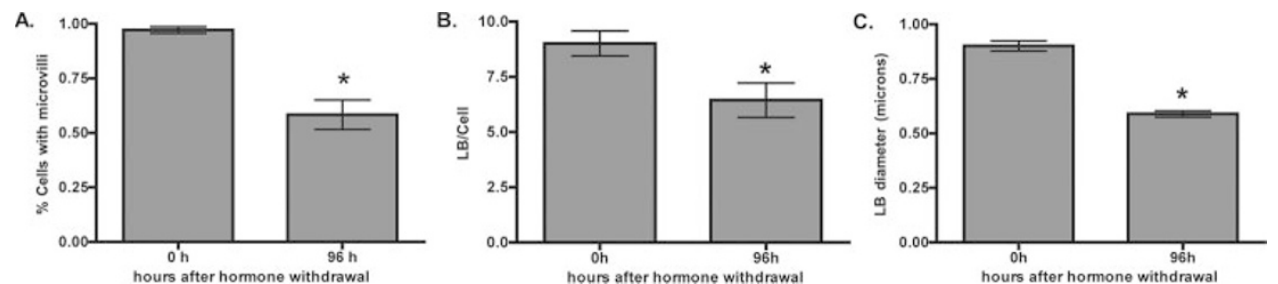

Figure 2. Quantitative morphology changes with transdifferentiation. TEM samples from three experiments were evaluated at $\times 5000$ after $4 \mathrm{~d} D C I(0 \mathrm{~h})$ and $96 \mathrm{~h}$ after hormone withdrawal from $4 \mathrm{~d}$ DCI-treated cells ( $4 \mathrm{~d}$ DCI $/ 4 \mathrm{~d}$ Waymouth's). (A) Percentage of cells exhibiting microvilli $\left({ }^{*} p<0.001\right)$. $(B)$ Lamellar bodies per cell $(* p<0.05 v s 4 \mathrm{~d}$ DCI). (C) Lamellar body diameter $(p<0.001)$.

strated fewer lamellar bodies and blunting of microvilli by TEM (Fig. 1B), despite maintaining a confluent monolayer (Fig. 3D). This was evidenced on SEM as a loss of surface projections (Fig. 1F).

Field counting supported these changes [three experiments, seven to eight high-power fields (hpf) per treatment group with $6.0 \pm 0.5$ cells/hpf]. Of $4 \mathrm{~d}$ DCI cells, $97 \pm 0.1 \%$ had microvilli. Withdrawal of hormones was associated with only $58.4 \pm 0.1 \%$ of cells exhibiting microvilli (Fig. $2 A)(p<$ 0.001 ). Lamellar bodies were also diminished (Fig. $2 B$ ) after hormone withdrawal $(6.4 \pm 0.8$ lamellar bodies/cell), compared with $4 \mathrm{~d}$ DCI type II controls $(9.0 \pm 0.6$ lamellar bodies/cell) $(p<0.05)$. Remaining lamellar bodies after hormone withdrawal were smaller (Fig. $2 C$ ), with a mean diameter of $0.6 \pm 0.01 \mu \mathrm{m}$ versus $0.9 \pm 0.02 \mu \mathrm{m}$ in $4 \mathrm{~d}$ DCI cells $(p<0.001)$. Together, these data show that transdifferentiation is associated with diminution of type II cell morphology.

Alveolar epithelial cell barrier function. We assessed epithelial barrier function by dye filtration and transepithelial resistance (Fig. 3). The concentration of both carboxyfluorescein (Fig. 3A) and Texas Red dextran (Fig. 3B) filtered into the bottom chamber was decreased in cells after hormone withdrawal $(n=9-11, p<0.05$ versus $4 \mathrm{~d}$ and $7 \mathrm{~d} \mathrm{DCI})$. Rat type II cells routinely demonstrate TER of 500-1000 $\Omega \times \mathrm{cm}^{2}$ (17). Human differentiated type II cells (4 d DCI) demonstrated a mean TER of $347 \pm 14 \Omega \times \mathrm{cm}^{2}$ (Fig. 3C). Mean TER increased with transdifferentiation, to $446 \pm 20 \Omega \times \mathrm{cm}^{2}$ ( $n=8, p<0.01$ versus $4 \mathrm{~d}$ and $7 \mathrm{~d}$ DCI) $3 \mathrm{~d}$ after hormone withdrawal. Together, these data indicate tighter barrier function with transdifferentiation.

Disappearance of type II cell markers. Real-time RT-PCR (Fig. 4A) revealed significant reduction of type II markers after hormone withdrawal. All markers were decreased to less than $50 \%$ of controls within $24 \mathrm{~h}(n=6-7, p<0.01$ versus $4 \mathrm{~d} \mathrm{DCI}$ ) and continued to decline throughout the subsequent $5 \mathrm{~d}(n=3-7, p<0.01$ versus $4 \mathrm{~d}$ DCI, except SP-B at $144 \mathrm{~h}$, for which $p<0.05$ ). Only a small amount of SP-B mRNA was present in nonhormone-treated controls (d 4 Waymouth's) (6.4 $\pm 2.3 \%$ of $d 4$ DCI values). Similar to our previous work (2), immunoblotting revealed that hormone withdrawal resulted in progressive decline of all type II markers studied, with disappearance of SP-A, NPro SP-C, and PGC by $72 \mathrm{~h}$ and SP-B by $120 \mathrm{~h}$ (Fig. $4 B$ ).

Increased expression of type I cell markers. Real-time RT-PCR (Fig. 5A) revealed expression of all type I markers examined in $4 \mathrm{~d}$ Waymouth's-treated controls, particularly PAI-1. Expression of the type I cell markers Cav-1 (mean $2.3 \pm 0.4$-fold), aquaporin 5 (mean $2.7 \pm 0.6$-fold), and PAI-1 (mean $5.1 \pm 0.7$-fold) increased with transdifferentiation over 24-96 h after hormone withdrawal, compared with type II controls $(n=7, p<0.05)$. There was no induction of claudin 7 mRNA. At $144 \mathrm{~h}$ after hormone withdrawal, PAI-1 remained significantly induced at $4.3 \pm 1.3$-fold versus $4 \mathrm{~d}$ DCI $(n=3, p<0.05)$. By immunoblotting, there were increases in the type I markers Cav-1 and PAI-1, with no change in claudin 7 (Fig. 5B). Although PAI-1 protein appeared earlier than Cav-1, both were induced by $72 \mathrm{~h}$ after hormone withdrawal. The type I cell marker aquaporin 5, induced in our mRNA studies, was also probed via immunoblotting, but only nonspecific banding patterns were visible using a variety of commercial antibodies (data not shown).
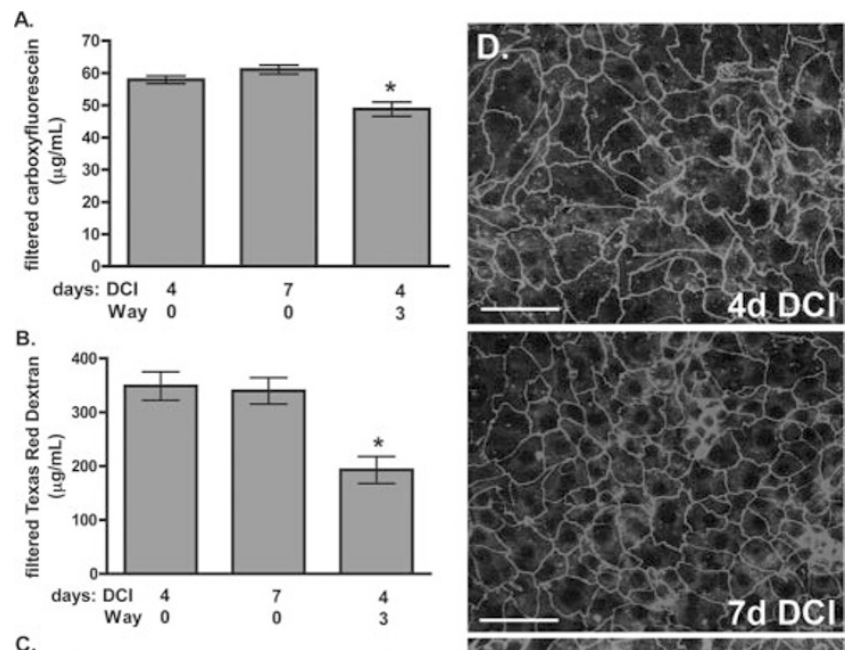

c.
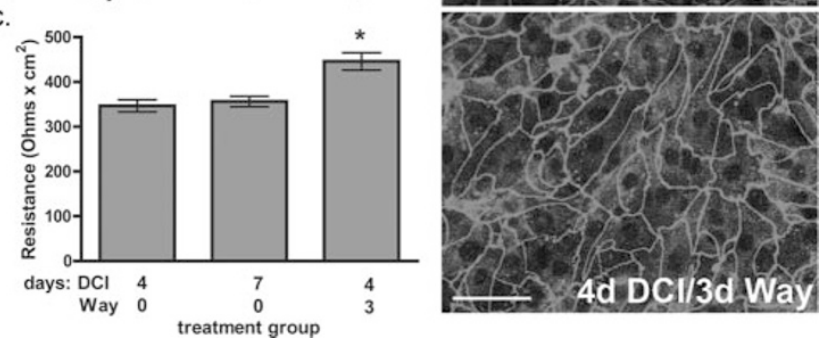

Figure 3. Increased barrier function with transdifferentiation. Epithelial barrier function was examined in cells cultured on permeable supports with $4 \mathrm{~d}$ DCI, $7 \mathrm{~d}$ DCI, or $4 \mathrm{~d}$ DCI followed by hormone withdrawal (4 d DCI/3 d Waymouth's). (A) Carboxyfluorescein filtration $(* p<0.05)$. (B) Texas Red dextran filtration $(* p<0.05)$. (C) TER $(* p<0.01)$. (D) Cells immunostained for ZO-1 at $\times 20$ demonstrate confluent monolayers. Bars $=20 \mu \mathrm{m}$. 
A.

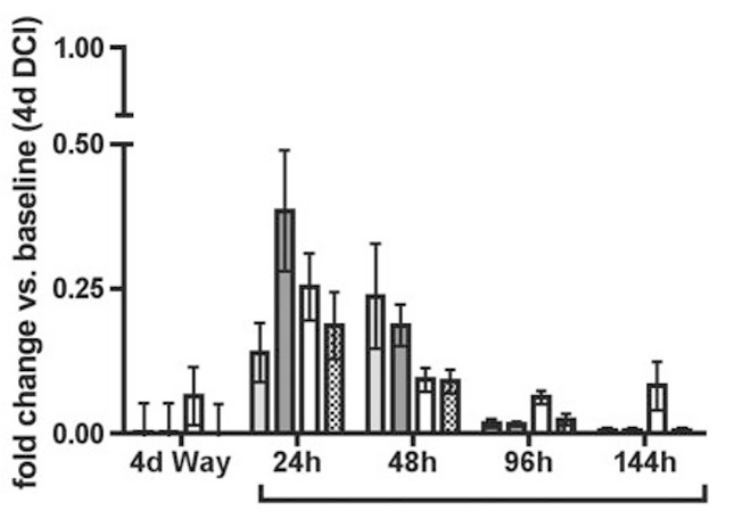

B.

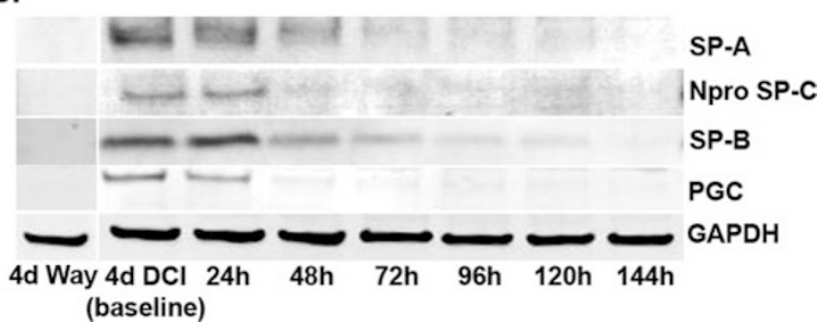

Figure 4. Type II cell markers decrease with transdifferentiation. The type II cell markers SP-A, SP-C, SP-B, and PGC were assessed by real-time RT-PCR $(A)$ and immunoblotting $(B)$ for up to $144 \mathrm{~h}$ after hormone withdrawal from 4 d DCI-treated cells. SPA (lightly shaded columns), SP-C (darkly shaded columns), SP-B (open columns), PGC (hatched columns).

A.

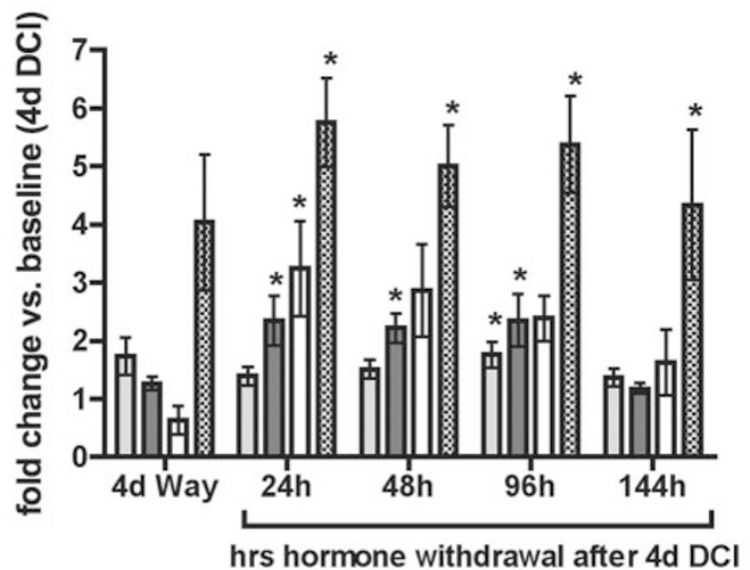

B.

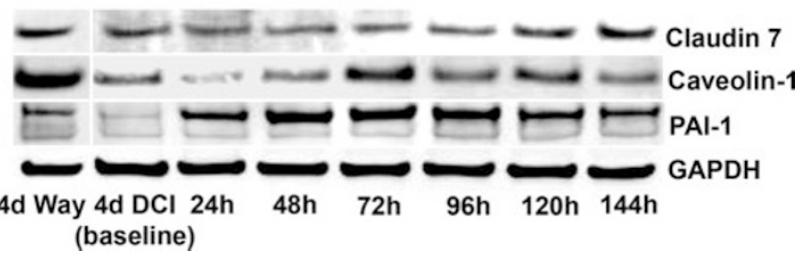

Figure 5. Type I cell markers increase with transdifferentiation. Type I cell markers claudin 7, Cav-1, aquaporin 5, and PAI-1 were assessed by real-time RT-PCR $(A)$ and immunoblotting $(B)$ for up to $144 \mathrm{~h}$ after hormone withdrawal from 4 d DCI-treated cells. Claudin 7 (lightly shaded columns), Dark gray: Cav-1 (darkly shaded columns), aquaporin 5 (open columns), PAI-1 (hatched columns) $(* p<0.05)$.

\section{DISCUSSION}

Difficulties in isolating and maintaining type I cells in culture, limited functional assays, and a paucity of cross- species reproducible markers have limited investigations of type I cell functions beyond gas exchange. Although investigators have used transdifferentiation as a model of adult type I cells $(9,18)$, this is the first report of transdifferentiation leading to a type I-like cell from human fetal alveolar epithelium. Transdifferentiated fetal cells lost type II cell characteristics. Importantly, transdifferentiated fetal cells demonstrated not only a functionally tight monolayer consistent with type I cell behavior, but increased expression of a battery of type I cell markers. The ability to elicit transdifferentiation in human fetal alveolar epithelium provides investigators with a unique opportunity to explore the mechanisms of fluid balance, epithelial cell lineage, and phenotype plasticity in the developing human lung.

Isolation and culture of type I cells has been difficult for several reasons. Type I cell numbers are decreased relative to type II cells (1), resulting in low yield. They require higher digestive enzyme concentrations to isolate them from surrounding matrix (9). Investigators have used techniques including tissue enzyme digestion, followed by density gradient centrifugation methods (9) or, alternatively, combinations of gradient centrifugation and magnetic beads to remove type II cells from type I cells (6). Such methods generally necessitated immediate analysis of the isolated type I cells. There are few reports of long-term culture of type I cells (5). This has prompted searches for alternative models. Most popular has been a model of transdifferentiation, based on the repeated observation that adult type II cells on tissue culture plastic lose the type II cell phenotype (19) and gain type I cell markers $(3,10)$. The model outlined in this report is the first description of successfully applying this technique to human fetal cells.

Poor functional markers have also complicated studies of type I cell biology. Although recent reports suggested that type I cells are an important source of antioxidants $(20,21)$, two established physiologic functions of the type I cell are gas exchange and serving as a barrier to fluid transport. Barrier function has been evaluated by characterizing ion and water channels $(22,23)$, micromolecular permeability (24), transepithelial resistance (8), and electrophysiologic measurement of ion channel activity (25). Claudins are an important mediator of this barrier function (26). Human fetal lung expresses claudins $1,3,4,5,7$, and 18 (16), and changes in claudin expression and cellular localization correlate with changes in alveolar epithelial cell permeability (15). In our model, claudin 7 expression did not significantly change with transdifferentiation. The observed increase in barrier function may be mediated by changes in claudin 7 localization or by other claudins and/or junctional proteins not yet examined. It is important to note that the observed tighter alveolar epithelial barrier with transdifferentiation concurs with rat transdifferentiation models (27). Although our measured TERs were lower than in the rat studies, they are similar to TER measurements from cultured human airway epithelial cells (28).

Studies of type I cell biology have been hampered by a lack of reliable markers that distinguish type I cells from type II cells. Recently, newer specific type I cell markers have come from adult rodent $(9,18)$ and adult human (29) models. In our studies, multiple type I markers were induced with transdif- 
ferentiation from the type II cell to the type I cell phenotype, with Cav-1 and PAI-1 being the most robust indicators of transdifferentiation. However, not all purported type I cell markers were induced in our model. Although we establish that a reliable panel of markers signifying a type I cell-like phenotype is expressed in human transdifferentiating cells, our results suggest that studies of type I cells should incorporate multiple markers as no single marker may accurately reflect changes in phenotype.

Cav-1 is a component of caveolae, plasma membrane invaginations that are lipid rich and involved in signaling, intracellular trafficking, and protein sorting (30). It exists in two isoforms, alpha and beta (31). In the mouse lung, the alpha isoform is present in both endothelium and type I epithelial cells. Our model concurs with other nonhuman models in which Cav-1 appears with transdifferentiation toward a type I cell-like phenotype $(10,18)$. However, our model differs with respect to two previous observations. Other investigators noted no Cav-1 $\alpha$ in fetal or neonatal alveolar epithelium (32), and no Cav-1 in adult rat type II cells (33). We observed expression of Cav- $1 \alpha$ in all treatment groups, albeit with the highest expression seen in the type I-like transdifferentiated cells. Although this may reflect species-specific differences, our studies suggest that fetal alveolar epithelium is more multipotential than previously suspected.

PAI-1 was the most induced type I cell marker in our model. It is a serine protease inhibitor participating in fibrinolysis in many tissues, including the lung. Hyperoxic injury up-regulates PAI-1 (34) in murine models and mice lacking the PAI-1 gene have a decreased fibrotic response to bleomycin (35). PAI-1 is elevated in preterm infants with respiratory distress syndrome (RDS) and may play a contributory role in bronchopulmonary dysplasia (36). The robust up-regulation of PAI-1 with transdifferentiation seen here supports the likely important role of PAI-1 in tissue repair in the alveolar microenvironment.

The transition between epithelial cell phenotypes in our model was not complete by the end of the culture period, and there was baseline expression of many markers by untreated control cells. With transdifferentiation, type II markers were still evident, albeit reduced to less than $10 \%$ of controls. The incompletely flattened appearance (Fig. 1D) of our transdifferentiated cells may be due to culture conditions and agrees with previous reports (3). Not all microvilli and lamellar bodies were lost by $96 \mathrm{~h}$, as in prior reports. Thus, transdifferentiated cells, although type I-like, have a somewhat intermediate phenotype. Work by others suggests differences between freshly isolated type I cells and transdifferentiated cells (21). It is important to acknowledge that transdifferentiation is an imperfect model for studying type I cells, but remains a powerful tool for initial investigations into type I cell biology, until more reliable primary culture methods for type I cells are available.

Our data have important implications regarding the developmental lineage of type II and type I cells. Evidence from adult animal models suggests that type I cells are derived from type II cells $(19,37)$, as type II cells are progenitors of type I cells after alveolar injury in mature lung (11) and isolated type
II cells in culture tend to lose characteristics of type II cells, adopting a more type I-like appearance (7). However, in vitro (38) and in vivo (39) evidence supports the plasticity of alveolar epithelial cell phenotype $(38,40)$. Our data support the prevailing concept that human fetal type II cells can give rise to type I-like cells. Our data also support the notion of epithelial cell plasticity; given the observation of some type I cell markers in our type II cells. However, our findings of type I cell markers with selected type II cell markers in untreated cells imply that our starting cell population is far from undifferentiated and advocates a role for greater plasticity in fetal alveolar progenitor epithelial cells than has been classically appreciated.

In summary, we have shown that under defined conditions, human fetal alveolar type II cells transdifferentiate, with regression of type II cell morphologic and phenotypic markers. Transdifferentiation is further associated with increased expression of type I cell markers and increased alveolar epithelial cell barrier function. This model will serve as a useful tool for further study of human fetal alveolar epithelial cell differentiation and type I cell biology.

Acknowledgments. The authors thank Sree Angampalli and Ping Wang for type II cell preparation, Michael Koval for assistance with the barrier studies, and Neelimah Shah at the Biomedical Imaging Core of the University of Pennsylvania School of Medicine for the microscopy images. They also thank Michael Beers and Harry Ischiropoulos for editorial assistance.

\section{REFERENCES}

1. Mallampalli RK, Acarregui MJ, Snyder JM 1997 Differentiation of the alveolar epithelium in the fetal lung. In: McDonald JA (ed) Lung Growth and Development. Marcel Dekker, New York, pp 119-162

2. Foster C, Aktar A, Kopf D, Zhang P, Guttentag S 2004 Pepsinogen C: a type 2 cell-specific protease. Am J Physiol Lung Cell Mol Physiol 286:L382-L387

3. Borok Z, Hami A, Danto SI, Zabski SM, Crandall ED 1995 Rat serum inhibits progression of alveolar epithelial cells toward the type I cell phenotype in vitro. Am J Respir Cell Mol Biol 12:50-55

4. Williams MC 2003 Alveolar type i cells: molecular phenotype and development. Annu Rev Physiol 65:669-695

5. Chen J, Chen Z, Narasaraju T, Jin N, Liu L 2004 Isolation of highly pure alveolar epithelial type I and type II cells from rat lungs. Lab Invest 84:727-735

6. Dobbs LG, Gonzalez R, Matthay MA, Carter EP, Allen L, Verkman AS 1998 Highly water-permeable type I alveolar epithelial cells confer high water permeability between the airspace and vasculature in rat lung. Proc Natl Acad Sci U S A 95:2991-2996

7. Mason RJ, Dobbs LG 1980 Synthesis of phosphatidylcholine and phosphatidylglycerol by alveolar type II cells in primary culture. J Biol Chem 255:5101-5107

8. Chen SP, Zhou B, Willis BC, Sandoval AJ, Liebler JM, Kim KJ, Ann DK, Crandall ED, Borok Z 2005 Effects of transdifferentiation and EGF on claudin isoform expression in alveolar epithelial cells. J Appl Physiol 98:322-328

9. Dobbs LG, Williams MC, Gonzalez R 1988 Monoclonal antibodies specific to apical surfaces of rat alveolar type I cells bind to surfaces of cultured, but not freshly isolated, type II cells. Biochim Biophys Acta 970:146-156

10. Fuchs S, Hollins AJ, Laue M, Schaefer UF, Roemer K, Gumbleton M, Lehr CM 2003 Differentiation of human alveolar epithelial cells in primary culture: morphological characterization and synthesis of caveolin-1 and surfactant protein-C. Cell Tissue Res 311:31-45

11. Evans MJ, Cabral LJ, Stephens RJ, Freeman G 1975 Transformation of alveolar type 2 cells to type 1 cells following exposure to NO2. Exp Mol Pathol 22:142-150

12. Gonzales LW, Angampalli S, Guttentag SH, Beers MF, Feinstein SI, Matlapudi A, Ballard PL 2001 Maintenance of differentiated function of the surfactant system in human fetal lung type II cells cultured on plastic. Pediatr Pathol Mol Med 20:387_ 412

13. Gonzales LW, Guttentag SH, Wade KC, Postle AD, Ballard PL 2002 Differentiation of human pulmonary type II cells in vitro by glucocorticoid plus cyclic AMP. Am J Physiol Lung Cell Mol Physiol 283:L940-L951

14. Foster CD, Zhang PX, Gonzales LW, Guttentag SH 2003 In vitro surfactant protein B deficiency inhibits lamellar body formation. Am J Respir Cell Mol Biol 29:259266 
15. Wang F, Daugherty B, Keise LL, Wei Z, Foley JP, Savani RC, Koval M 2003 Heterogeneity of claudin expression by alveolar epithelial cells. Am J Respir Cell Mol Biol 29:62-70

16. Daugherty BL, Mateescu M, Patel AS, Wade K, Kimura S, Gonzales LW, Guttentag S, Ballard PL, Koval M 2004 Developmental regulation of claudin localization by fetal alveolar epithelial cells. Am J Physiol Lung Cell Mol Physiol 287:L1266L1273

17. Cheek JM, Evans MJ, Crandall ED 1989 Type I cell-like morphology in tight alveolar epithelial monolayers. Exp Cell Res 184:375-387

18. Qiao R, Zhou B, Liebler JM, Li X, Crandall ED, Borok Z 2003 Identification of three genes of known function expressed by alveolar epithelial type I cells. Am J Respir Cell Mol Biol 29:98-105

19. Adamson IY, Bowden DH 1975 Derivation of type 1 epithelium from type 2 cells in the developing rat lung. Lab Invest 32:736-745

20. Chen J, Chen Z, Chintagari NR, Bhaskaran M, Jin N, Narasaraju T, Liu L 2006 Alveolar type I cells protect rat lung epithelium from oxidative injury. J Physiol 572:625-638

21. Gonzalez R, Yang YH, Griffin C, Allen L, Tigue Z, Dobbs L 2005 Freshly isolated rat alveolar type I cells, type II cells, and cultured type II cells have distinct molecular phenotypes. Am J Physiol Lung Cell Mol Physiol 288:L179-L189

22. Kemp PJ, Kim KJ 2004 Spectrum of ion channels in alveolar epithelial cells: implications for alveolar fluid balance. Am J Physiol Lung Cell Mol Physiol 287:L460-L464

23. Matthay MA, Folkesson HG, Verkman AS 1996 Salt and water transport across alveolar and distal airway epithelia in the adult lung. Am J Physiol 270:L487L503

24. Cavanaugh KJ, Cohen TS, Margulies SS 2006 Stretch increases alveolar epithelial permeability to uncharged micromolecules. Am J Physiol Cell Physiol 290:C1179C1188

25. Bourke S, Mason HS, Borok Z, Kim KJ, Crandall ED, Kemp PJ 2005 Development of a lung slice preparation for recording ion channel activity in alveolar epithelial type I cells. Respir Res 6:40

26. Van Itallie CM, Anderson JM 2004 The molecular physiology of tight junction pores. Physiology (Bethesda) 19:331-338

27. Borok Z, Hami A, Danto SI, Lubman RL, Kim KJ, Crandall ED 1996 Effects of EGF on alveolar epithelial junctional permeability and active sodium transport. Am J Physiol 270:L559-L565
28. Everett RS, Vanhook MK, Barozzi N, Toth I, Johnson LG 2006 Specific modulation of airway epithelial tight junctions by apical application of an occludin peptide. Mol Pharmacol 69:492-500

29. Newman V, Gonzalez RF, Matthay MA, Dobbs LG 2000 A novel alveolar type cell-specific biochemical marker of human acute lung injury. Am J Respir Crit Care Med 161:990-995

30. Anderson RG 1998 The caveolae membrane system. Annu Rev Biochem 67:199225

31. Kogo H, Fujimoto T 2000 Caveolin-1 isoforms are encoded by distinct mRNAs Identification Of mouse caveolin-1 mRNA variants caused by alternative transcription initiation and splicing. FEBS Lett 465:119-123

32. Ramirez MI, Pollack L, Millien G, Cao YX, Hinds A, Williams MC 2002 The alpha-isoform of caveolin-1 is a marker of vasculogenesis in early lung development. J Histochem Cytochem 50:33-42

33. Campbell L, Hollins AJ, Al-Eid A, Newman GR, von Ruhland C, Gumbleton M 1999 Caveolin-1 expression and caveolae biogenesis during cell transdifferentiation in lung alveolar epithelial primary cultures. Biochem Biophys Res Commun 262:744-751

34. Barazzone C, Belin D, Piguet PF, Vassalli JD, Sappino AP 1996 Plasminogen activator inhibitor-1 in acute hyperoxic mouse lung injury. J Clin Invest 98:2666-2673

35. Eitzman DT, McCoy RD, Zheng X, Fay WP, Shen T, Ginsburg D, Simon RH 1996 Bleomycin-induced pulmonary fibrosis in transgenic mice that either lack or overexpress the murine plasminogen activator inhibitor-1 gene. J Clin Invest 97:232-237

36. Cederqvist K, Siren V, Petaja J, Vaheri A, Haglund C, Andersson S 2006 High concentrations of plasminogen activator inhibitor-1 in lungs of preterm infants with respiratory distress syndrome. Pediatrics 117:1226-1234

37. Diglio CA, Kikkawa Y 1977 The type II epithelial cells of the lung. IV. Adaption and behavior of isolated type II cells in culture. Lab Invest 37:622-631

38. Danto SI, Shannon JM, Borok Z, Zabski SM, Crandall ED 1995 Reversible transdifferentiation of alveolar epithelial cells. Am J Respir Cell Mol Biol 12:497-502

39. Flecknoe SJ, Wallace MJ, Harding R, Hooper SB 2002 Determination of alveola epithelial cell phenotypes in fetal sheep: evidence for the involvement of basal lung expansion. J Physiol 542:245-253

40. Borok Z, Danto SI, Lubman RL, Cao Y, Williams MC, Crandall ED 1998 Modulation of t1alpha expression with alveolar epithelial cell phenotype in vitro. Am J Physiol 275:L155-L164 